\title{
Percepção de velocidade do movimento biológico: mais resistente ao fenômeno de interferência?!
}

\author{
Perception of speed in biological motion: \\ more resistant to interference?
}

\author{
Sandra MOUTA M $^{2,3}$ \\ Jorge de Almeida SANTOS ${ }^{4}$
}

\begin{abstract}
Resumo
O sistema visual humano é frequentemente referido como altamente preparado para extrair informação relevante de padrões de movimento biológico. Nesse sentido, este estudo analisa o efeito de contraste na percepção de velocidade. Os participantes realizaram o julgamento de velocidade numa situação na qual dois point-light walkers simultâneos foram apresentados com diferentes contrastes relativamente ao fundo e com diferentes velocidades de translação. Na Experiência 1, o movimento de translação biológico canônico foi comparado com o movimento de translação rígido, enquanto na Experiência 2 ele foi comparado com o movimento de translação biológico invertido. O padrão biológico canônico apresenta maior taxa de erro, tempos de reação mais elevados e maior vulnerabilidade ao efeito de contraste na percepção da velocidade do que o padrão rígido. No entanto, não foram encontradas diferenças significativas entre o estímulo canônico e o invertido. A Experiência 3 foi implementada com o objetivo de se controlar o papel das pistas posicionais na tarefa de julgamento de velocidade. Os pontos iniciais e finais da trajetória foram combinados de modo a que os point-light walkers mais rápidos e os mais lentos pudessem terminar o ensaio numa posição relativamente mais avançada ou atrasada. Apesar desta variação, o padrão de resultados foi congruente com as observações das Experiências 1 e 2. Aparentemente, os participantes realizaram julgamentos de velocidade factuais, ao invés do uso de pistas espaciais como uma espécie de referência ou comparação de posicionamento. Dado que a percepção dos padrões biológicos foi mais vulnerável aos efeitos de contraste, mas não foi afetada pela familiaridade, este estudo sugere que a percepção de movimento biológico e rígido poderá obedecer às mesmas regras computacionais, pelo menos em tarefas que envolvam padrões em translação e julgamentos de velocidade.
\end{abstract}

Unitermos: Efeitos de contraste. Percepção visual. Movimento.

\begin{abstract}
The human visual system is often referred to as being highly prepared to extract meaningful information from biological motion patterns. In the present study, the contrast effect on speed perception was analysed. Participants performed a test of speed judgment in which two simultaneous point-light walkers were animated at different translational speeds and contrasts in relation to the background. Standard translational biological motion was compared to rigid translational motion in Experiment 1 and to inverted biological motion in Experiment 2. Higher error rates, reaction times and greater vulnerability to contrast effects on speed perception were found for translational biological

$\boldsymbol{\nabla} \mathbf{v} \boldsymbol{\nabla}$

I Apoio: Fundação para a Ciência e Tecnologia, SFRH/BD/18265/2004; REEQ/821/PSI/2005.

2 Universitat de Barcelona, Departamento de Psicologia Bàsica, Vision \& Control of Action Group. Catalonia, España.

3 Universidade do Porto, Instituto de Engenharia de Sistemas e Computadores. Campus da FEUP, R. Dr. Roberto Frias, 378, $4200-465$ Porto, Portugal. Correspondência para/Correspondence to: S. MOUTA. E-mail: <smouta@psi.uminho.pt>.

4 Universidade do Minho, Centro de Computação Gráfica, Departamento de Psicologia Básica \& Centro Algoritmi. Guimarães, Portugal.
\end{abstract}


motion when compared to rigid motion. No significant differences were found, however, between standard and inverted stimuli. Experiment 3 was implemented in order to control the role of positional cues in speed judgment. The start and finish points of the trajectory were varied so that the faster and slower point-light walkers could finish the trial at a relatively more advanced or more withdrawn position. In spite of the variation of the start and finish points of the trajectories, the pattern of results was still consistent with the findings of Experiments 1 and 2. Participants seemed to perform factual speed judgments instead of using spatial cues as a form of reference or positional matching. Since the perception of biological patterns was more sensitive to contrast effects and not affected by familiarity, it is suggested that perception of biological and rigid motion may follow the same computational rules, at least for tasks involving translational patterns and speed judgment.

Uniterms: Contrast Effects. Visual perception. Motion

Desde os estudos de Johansson (1973), a percepção de movimento biológico tem sido recorrentemente apontada como um dos maiores exemplos da robustez do sistema visual humano. O estímulo mais utilizado nos estudos de percepção de movimento biológico é o Point-Light Walker (PLW), que consiste num conjunto de pontos que representam as principais articulações do corpo humano, sendo qualquer outro tipo de informação estrutural removida. Quando esses estímulos são apresentados de forma estática, o seu reconhecimento é praticamente nulo; porém, com animação representativa de movimento, as taxas de reconhecimento elevam-se para valores próximos dos 100\% (Johansson, 1973). Esse fenômeno é válido não só para a identificação do movimento, mas também para a identificação de ações (Dittrich, 1993; Norman, Payton, Long \& Hawkes, 2004), gênero (Pollick, Kay, Heim \& Stringer, 2005; Troje, Sadr, Geyer \& Nakayama, 2006), emoções (Atkinson, Dittrich, Gemmell \& Young, 2004; Pollick, Paterson, Bruderlin \& Sanford, 2001), identidades específicas (Westhoff \& Troje, 2007), ou até mesmo o padrão de marcha do próprio observador (Beardsworth \&Buckner, 1981).

Estudos que utilizaram ruído temporal e espacial referem que a detecção de movimento biológico é altamente resistente ao uso de máscara (Bertenthal \& Pinto, 1994; Cutting, Moore \& Morrison, 1988; Thompson, Hansen, Hess \& Troje, 2007). Esse tipo de deterioração do estímulo normalmente faz aumentar a janela temporal para execução da tarefa, mas não diminui os elevados níveis de detecção do movimento biológico. Todos esses estudos sugerem que o sistema visual humano está altamente preparado para extrair informação significativa, de uma forma rápida e eficiente, a partir do movimento biológico. Sugerem também que esse fato é válido mesmo para estímulos empobrecidos, como é o caso de PLW, e sujeitos a diferentes tipos de ruído. No 476 entanto, a sensibilidade para a detecção de movimento biológico relaciona-se diretamente com a familiaridade do estímulo e da sua perspectiva. Quando PLW semeIhantes aos utilizados nos estudos anteriores são apresentados de uma forma invertida (com rotação de $180^{\circ}$ no eixo horizontal), a informação estrutural e a complexidade do padrão de movimento mantêm-se. Contudo, os níveis de reconhecimento diminuem de uma forma drástica (Pavlova \& Sokolov, 2000; Sumi, 1984; Troje, 2003). Assim, os estímulos de movimento biológico invertido funcionam como um estímulo de controle, já que podem ser considerados estímulos não-biológicos com o mesmo nível de complexidade estrutural. Pode-se concluir que o reconhecimento do movimento biológico é um fenômeno robusto, mas baseado em mecanismos muito específicos e em pistas dependentes da perspectiva.

Até aqui foram referidos estudos que apontam o sistema visual humano como extremamente sensível no reconhecimento e detecção do movimento biológico, isto é, em tarefas de alto nível. Na maioria desses estudo, os PLW são apresentados durante a execução de um movimento em que a componente translacional comum é removida (por exemplo: num movimento de marcha, o estímulo é apresentado como se estivesse a andar numa passadeira). Parece importante estudar outros processos perceptivos mais básicos, mas importantes para tarefas quotidianas. A percepção de movimento translacional biológico é crítica no funcionamento perceptivo e motor do ser humano, tanto para evitar colisões quanto para executar tarefas de navegação ou interagir com outros seres vivos.

Por isso, este estudo tem como objetivo verificar se o fato de haver especial sensibilidade para a percepção de movimento biológico, tal como os estudos de reconhecimento indicam, se mantém em tarefas cujo padrão de movimento inclua uma componente de translação. Assim, será comparado o desempenho dos participantes numa tarefa de discriminação de velocidade 
entre padrões de movimento translacional rígido (não-biológico) e translacional biológico.

Neri, Morrone e Burr (1988) demonstram que tarefas como a detecção e discriminação da orientação de movimento biológico requerem maior integração temporal, quando comparadas com movimento translacional rígido. Note-se que, no estudo citado, a componente comum de translação foi também removida dos estímulos de movimento biológico. No presente estudo, se realmente a robustez da percepção do movimento biológico se mantiver, o desempenho nessa tarefa deverá ser mais rápido e/ou mais acertado perante padrões desse tipo de movimento. Por outro lado, se a percepção de padrões de movimento translacional, quer biológicos quer não-biológicos, respeitar as mesmas regras computacionais, o desempenho da tarefa para o movimento biológico deverá ser inferior, pois se trata de um movimento mais complexo, e por isso torna a tarefa mais exigente.

Um objeto rígido com movimento translacional possui, para todos os seus pontos, um vetor de velocidade semelhante que resulta numa componente de translação visual única. Esse fenômeno não acontece para o movimento biológico. Para além da componente translacional comum, esses padrões são constituídos por componentes rotativas e pendulares resultantes dos movimentos relativos de todos os segmentos do corpo (para uma análise detalhada consultar a abordagem vetorial de Johansson (1973). Casile e Giese (2005) defendem que o sistema visual humano executa uma análise independente das componentes horizontal e vertical do movimento biológico, realizada através de redes neuronais separadas e especializadas em diferentes direções de movimento. Em suma, os resultados dos estudos clássicos de reconhecimento que sugerem que a percepção de movimento biológico é altamente eficiente parecem não ser compatíveis com o fato de esse ser um padrão de movimento complexo e por isso muito mais exigente em termos perceptivos. Essa exigência parece ainda aumentar quando se trata de tarefas que impliquem movimento translacional e julgamentos de baixo nível, como a percepção de velocidade.

A interferência do contraste na percepção de velocidade de objetos rígidos é um fenômeno bastante estudado (Anstis, 2001; 2003; Stone \& Thompson, 1992). Esse efeito de contraste consiste no fato de, na presença de dois objetos movendo-se à mesma velocidade mas com diferentes contrastes relativamente a um fundo isoluminante, o objeto com maior contraste ser percebido como mais rápido (velocidade aparente).

Neste estudo, tem-se como objetivo testar o fenômeno da interferência do contraste numa tarefa de discriminação de velocidade, comparando padrões de movimento translacional biológico e não-biológico (rígido ou invertido), com diferentes níveis de complexidade. Seguindo a hipótese de que o movimento biológico é mais complexo em termos computacionais, pode-se esperar que o desempenho na tarefa de discriminação de velocidade para esse tipo de movimento seja mais sensível ao erro, e que esse padrão seja mais sensível à interferência do fundo. Em alternativa, e tendo em conta o fato já descrito da alta resistência do movimento biológico aos efeitos de máscara na presença de padrões complexos de fundo (Bertenthal \& Pinto, 1994; Cutting et al., 1988; Thornton, Pinto \& Shiffrar, 1998), pode-se esperar que o movimento biológico seja mais resistente ao efeito do contraste, quando comparado com outro tipo de movimento, tal como o rígido ou invertido.

Com o objetivo de perceber se a percepção de movimento biológico em translação é semelhante aos processos envolvidos no reconhecimento desse tipo de padrão, ou pelo contrário, se ela segue as mesmas regras da percepção de objetos rígidos, o presente estudo desenvolveu três experiências. O objetivo geral do estudo foi compreender como a percepção de padrões de movimento biológicos e não-biológicos é influenciada pelo contraste entre objeto e fundo. Nas experiências descritas neste estudo foi pedido aos participantes para identificar qual de dois estímulos simultâneos foi o mais rápido. Os estímulos diferiram entre si relativamente à velocidade e ao nível de contraste com o fundo. Na primeira experiência comparou-se o desempenho perante padrões de movimento com diferentes níveis de exigência computacional: um estímulo rígido em translação (um frame "congelado" do PLW) e um estímulo biológico canônico em translação (animado com os movimentos relativos entre os membros, característicos do movimento biológico). Na segunda experiência foram comparados dois padrões de movimento biológico com a mesma carga computacional, mas com diferentes níveis de reconhecimento: um 
estímulo biológico canônico e um invertido. Na terceira experiência testou-se o papel de pistas espaciais (pontos inicial e final da trajetória) sobre o desempenho na tarefa.

\section{Método}

\section{Experiência 1}

O principal objetivo da Experiência 1 foi comparar os efeitos do contraste entre objeto e fundo na percepção de velocidade entre dois padrões de movimento: rígido e biológico em movimento. O padrão biológico consistiu num PLW animado, com um movimento em translação correspondente a um ciclo de passada gerado a partir de uma captação de movimento real recolhido a 30 frames por segundo. O estímulo de movimento rígido foi gerado através da utilização de apenas um frame do PLW apresentado na condição anterior. Cada padrão de movimento foi apresentado em três condições, definidas pelas combinações entre as diferentes velocidades e os parâmetros de contraste objeto/fundo.

Se o sistema perceptivo for altamente especializado na percepção de movimento biológico, então os resultados psicofísicos esperados deverão demonstrar um melhor desempenho e menores efeitos do contraste para esse tipo de padrão de movimento, quando comparado com o movimento rígido.

\section{Participantes}

Cinco participantes realizaram a tarefa experimental deste estudo, cujos objetivos eram conhecidos por apenas um deles. Todos possuíam visão normal ou corrigida, confirmada pelo teste automático Ergovision (Essilor) e por testes complementares de cinética (acuidade visual dinâmica, com estímulos em movimento cuja direção, velocidade e frequência espacial variavam) e visão de contraste.

\section{Instrumentos}

As experiências foram apresentadas num computador Apple G5 Macintosh Dual Core, com processador a 2.3 GHz, 2.5 GB de RAM, e placa gráfica NVIDIA GeForce 6600 com 256 MB de VRAM. As imagens foram 478 visualizadas num monitor Sony GDM-FW900, com reso- lução espacial de 1400×1050 pixels (ângulo visual horizontal de $15.5^{\circ}$ ) e rácio de refrescamento de $90 \mathrm{~Hz}$. Os níveis de luminância e contraste foram controlados com fotômetros Minolta LS100 e Tektronix J1800 Series LumaColor.

Os padrões de movimento foram criados a partir da base de dados do Laboratório de Psicologia Experimental da Universidade Católica de Leuven (disponível em http://ppw.kuleuven.be/labexppsy/actiondb.htm). A informação utilizada incluía 13 coordenadas tridimensionais do corpo humano em marcha, adquiridas por um sistema de captação de movimento. O PLW foi gerado com um software de modelação (Maya), a partir das marcas das principais articulações do corpo humano. A animação da marcha foi criada frame a frame, durante um ciclo de marcha, resultando em 30 frames. Dekeyser, Verfaillie e Vanrie (2002) descrevem detalhadamente técnicas de desenvolvimento de PLW.

Os estímulos consistiram em dois PLW com $0.900^{\circ} \times 5.35^{\circ}$ numa perspectiva ortogonal, localizados $1.14^{\circ}$ acima e abaixo da linha horizontal média da tela, movendo-se da direita para a esquerda em todos os estímulos. Os pontos que representavam as articulações principais do corpo humano tinham $0.15^{\circ}$ (5X5 pixels). As margens da tela foram suavizadas cerca de $0.5^{\circ}, \mathrm{com}$ o intuito de evitar o uso do limite da imagem como pista de referência. A duração de cada estímulo foi de 1.5s.

O design experimental incluiu duas variáveis: o tipo de movimento e a combinação contraste/velocidade. Relativamente ao tipo de movimento, foram apresentadas duas condições: movimento rígido e biológico canônico, ambos em translação. O estímulo biológico canônico consistiu na utilização de 30 frames, correspondentes a um ciclo de marcha capturado a partir de movimento real, com uma amostragem de 30 frames na apresentação do estímulo (PLW animado com movimento de marcha e em translação). Já o estímulo rígido consistiu na utilização de apenas um frame respeitante ao ciclo de marcha (como se o PLW estivesse "congelado" e em translação). O frame utilizado correspondia àquele com maior amplitude em termos de configuração, i.e., o frame em que os membros estão mais afastados da linha transversa média do corpo durante o ciclo de marcha. Assim, na condição de movimento rígido, o padrão biológico foi eliminado, mantendo a informação estrutural do estímulo. 
Cada tipo de movimento foi apresentado em três condições definidas pelas combinações dos parâmetros de velocidade e contraste objeto/fundo. Os PLW eram apresentados simultaneamente com as velocidades de $2.7 \%$ s e $3 \%$, ou $3 \%$ s e $3.3 \%$ s. A diferença na velocidade de translação foi sempre de $10 \%$ relativamente à velocidade de referência de $3 \%$. Os estímulos de movimento biológico foram gerados a partir de um único ensaio de captação de movimento real e, consequentemente, a partir de uma única velocidade de translação. Assim, a velocidade dos PLW foi manipulada através da alteração artificial da velocidade de translação, não havendo correspondência entre cada uma das condições e o ensaio de captação real de movimento. Daí, a diferença de velocidade entre os dois PLW de cada estímulo foi mantida mínima (10\%), para prevenir potenciais artefatos.

O contraste objeto/fundo foi manipulado através da variação de luminância do fundo, que foi de 20 ou $200 \mathrm{~cd} / \mathrm{m}^{2}$, correspondente a 10\% (cinzento escuro) e 100\% (branco, máxima luminância conseguida), respectivamente. A luminância dos pontos do PLW foi de $2 \mathrm{~cd} / \mathrm{m}^{2}$ (preto). Dado que o o estímulo era composto por uma grande área de fundo relativamente ao objeto-alvo (PLW), a luminância média foi semelhante à luminância do fundo. Devido a isso, os estímulos apresentados na experiência foram caracterizados pelo contraste de Weber (Anstis, 2001; Pelli, 1990), calculado da seguinte forma:

Weber contrast $=\mathrm{dL} / \mathrm{L}$ (Fórmula 1)

Weber contrast $=($ Lmax $-\operatorname{Lmin}) / L \min )($ Fórmula 2)

Onde Lmax é a luminância do fundo, e Lmin é a luminância do PLW. O contraste de Weber entre fundo e objeto foi de 9 para a combinação de $2-20 \mathrm{~cd} / \mathrm{m}^{2}$ (1-10\%) e 99 para a combinação de 2-200cd/m² (1-100\%).

O contraste objeto-fundo dos dois PLW apresentados no mesmo estímulo pode ser o mesmo (igual a 9 ou a 99) ou diferente (um igual a 9, e o outro igual a 99). A combinação contraste/velocidade foi manipulada em três condições experimentais - Neutra, em que os dois PLW não diferem no contraste; Congruente, em que os PLW diferem no contraste de forma congruente com a diferença de velocidade; e Incongruente, em que os PLW diferem no contraste de forma incongruente com a diferença de velocidade. Essa terminologia foi baseada de acordo com os resultados indicados por Stone eThompson (1992). Por exemplo, a condição Congruente correspondia ao PLW mais rápido com o nível de contraste mais elevado, e a condição Incongruente, ao PLW mais rápido com o menor nível de contraste.

A posição (superior e inferior) dos dois PLW foi contrabalanceada ao longo dos ensaios. Os estímulos apresentados nesta experiência foram desenvolvidos e validados por estudos-piloto.

\section{Procedimentos}

O estudo foi aprovado pelo Conselho Científico do Instituto de Educação e Psicologia da Universidade do Minho, Portugal, no âmbito do programa de doutoramento do primeiro autor. Todos os participantes assinaram um Termo de Consentimento livre e esclarecido antes de sua inclusão na amostra. Nas subtarefas experimentais foram utilizados o método de estímulo constante e o paradigma de escolha forçada entre duas alternativas. Foi pedido aos participantes que executassem, o mais depressa e corretamente possível, uma tarefa de discriminação de velocidade, consistente em indicar qual dos PLW apresentados simultaneamente era o mais rápido.

Os participantes sentaram-se a $1.5 \mathrm{~m}$ da tela do computador, numa sala escura, segurando o mouse na mão dominante. A tarefa foi executada pressionando ou botão direito do mouse, se o PLW mais rápido fosse o superior; ou o botão esquerdo, se fosse o PLW mais rápido fosse o inferior. Cada participante realizou um período de treino, em que executou vinte avaliações de velocidade de uma condição neutra (sem combinação contraste/velocidade).

A experiência foi constituída por quatro blocos de 100 ensaios para cada um dos tipos de movimento movimento em translação rígido e movimento biológico. Em cada bloco, as três combinações de velocidade/contraste (Neutra, Congruente e Incongruente) foram apresentadas numa ordem quase aleatória. Cada estímulo foi apresentado durante 1 segundo (s), com um frame rate de $30 \mathrm{HZ}$. O intervalo interestímulos (IIE) tinha também a duração de 1s e consistia na apresentação de uma cruz (+) central, para que a localização da fixação ocular fosse mantida no início de todos os estí- 
mulos. A resposta dos participantes poderia ocorrer durante a apresentação do estímulo e no IIE. A percentagem de respostas corretas e o tempo de reação foram calculados para cada tipo de movimento e combinação de condições.

\section{Resultados}

A análise de dados exploratória revelou a ausência de missing values e de qualquer viés de posição, ou seja, não foi encontrada preferência pela posição do PLW, inferior (48\%) ou superior (52\%). Além disso, como não foram encontradas diferenças no desempenho entre os dois pares de valores de velocidade (2.7 vs.3.0 e 3.0 vs. $3.3 \mathrm{~m} / \mathrm{s}$ ), os resultados dessas condições foram agrupados nas análises subsequentes. Todos os resultados apresentaram uma distribuição normal, indicada pelo teste de normalidade de Kolmogorov-Smirnov, com a correção de significância de Lilliefors. Com o intuito de verificar a ocorrência de diferenças significativas na distribuição dos dados, foram executadas análises intersujeito e item a item. Os resultados de uma one-way ANOVA demonstraram não existir efeito da variável participante na análise de cada condição. Assim, foi decidido substituir o número de participantes pelo número de blocos de ensaios como unidade de análise (passou-se de $\mathrm{N}=5$ para $\mathrm{N}=20$ ).

\section{Acuidade}

A Figura 1 apresenta a percentagem de respostas corretas (média entre todos os participantes e Desvio-Padrão - DP) para cada combinação de velocidade/ contraste e tipo de movimento. O General Linear Model para medidas repetidas demonstrou fortes efeitos dos dois fatores de análise - combinação de velocidade/ contraste $[F(2,38)=28.98, p<0,001]$ e tipo de movimento $[F(1,19)=14,80, p<0,001]$. Não foi encontrado efeito de interação entre os dois fatores $[F(2,38)=1,23$, n.s.]. Foram realizadas Pairwise combinations através do ajustamento Bonferroni para múltiplas comparações.

Os resultados sugerem dois efeitos. Os julgamentos de velocidade são mais acertados na presença do movimento rígido, quando comparado com movimento biológico; e mais acertados na condição Con480 gruente, quando comparada com a condição Incon-

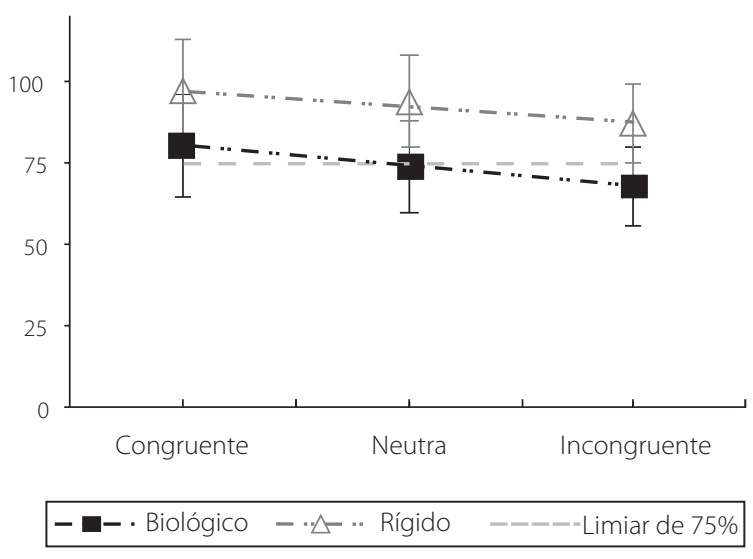

Figura 1. Percentagem média de respostas corretas em função da combinação Velocidade/Contraste para cada padrão de movimento, na Experiência 1. Braga (Portugal), 2008.

gruente. Para o movimento biológico, apenas se obtiveram valores superiores ao limiar de $75 \%$ na condição Congruente [média(DP). 93(16), 80(14) e 63(12), respectivamente para as condições Congruente, Neutra e Incongruente], enquanto para o movimento rígido foram obtidos valores superiores a esse valor em todas as combinações velocidade/contraste [média(DP). 100(4), 98(9) e 90(9), para as condições Congruente, Neutra e Incongruente respectivamente].

Os resultados médios são compatíveis com os dados individuais, já que todos os participantes apresentaram padrões de resultados semelhantes.

\section{Tempo de reação}

O tempo de reação requerido para o desempenho da tarefa na presença do movimento biológico foi superior ao tempo requerido para o movimento rígido (Figura 2). Os participantes levaram em média 1200 ms para responder ao primeiro [média(SD). 1192(87,89), 1231 (99,86) e 1244(86,99), para as condições Congruente, Neutra e Incongruente respectivamente]., e cerca de $1000 \mathrm{~ms}$ para responder ao segundo, que éa duração de apresentação do estímulo [média(SD). 998.17(222.19), 1002.16(210.43) e 1024.94(200.95), para as condições Congruente, Neutra e Incongruente, respectivamente]. Verificou-se um ligeiro aumento no tempo de reação da condição Congruente para a condição Incongruente. Uma ANOVA para medidas repetidas 


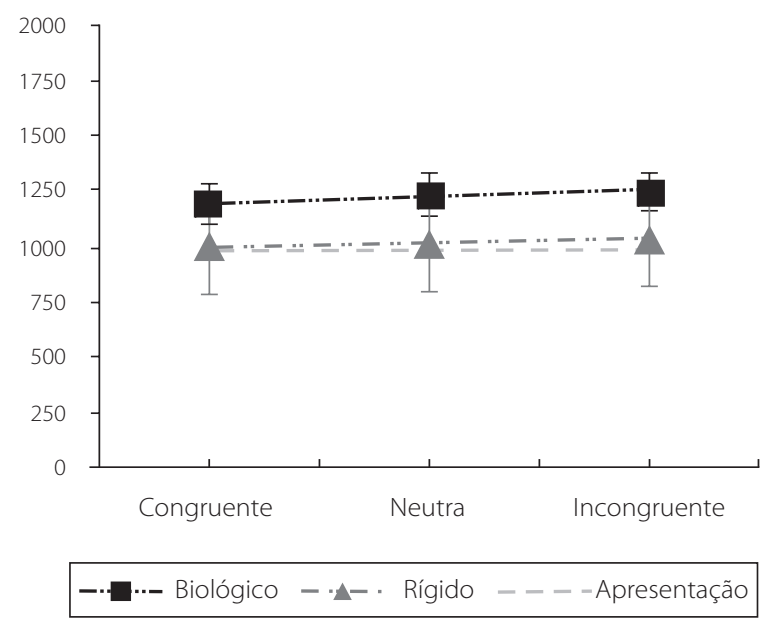

Figura 2. Tempo de reação médio (milissegundos) em função da combinação Velocidade/Contraste para cada padrão de movimento, na Experiência 1. Braga (Portugal), 2008.

indicou um efeito significativo do tipo de movimento $[F(1,19)=55,43, p<0,01]$, mas não da condição velocidade/ contraste $[F(2,38)=7594$, n.s.], nem efeitos de interação entre os dois fatores $[F(2,46)=3907$, n.s.]. Individualmente, todos os participantes responderam mais rapidamente na pre-sença do estímulo rígido.

\section{Discussão}

A primeira hipótese deste estudo propôs que o desempenho do sistema visual perante o movimento rígido ou o biológico (complexo) deveria ser significativamente diferente. A segunda hipótese postulada foi de que o contraste entre o objeto e o fundo deveria ter diferentes efeitos sobre a percepção de velocidade para cada um dos tipos de movimento. Nesta experiência, foi pedido que os participantes identificassem qual de dois estímulos simultâneos se movia mais rapidamente. Os estímulos diferiam na velocidade e no nível de contraste com o fundo. Foram apresentados dois tipos de movimento: rígido e biológico.

Os resultados são consistentes com os resultados de estudos prévios acerca do efeito do contraste na percepção de velocidade de objetos rígidos. Stone e Thompson (1992) demonstraram que, quando o contraste do objeto de referência é maior, a velocidade do estímulo de comparação é subestimada em cerca de
14 pontos percentuais; e, inversamente, quando o contraste do objeto de referência é menor, a velocidade do estímulo de comparação é sobrestimada em 17 pontos percentuais. De sublinhar que esses resultados foram obtidos para estímulos simultâneos movendo-se à mesma velocidade; portanto, os julgamentos foram baseados na percepção de velocidade aparente.

Partindo desse efeito de contraste na percepção de velocidade, seria esperado que a percentagem de respostas corretas aumentasse na condição Congruente (PLW mais rápido com maior contraste) e diminuísse na condição Incongruente (PLW mais rápido com menor contraste), quando comparadas com o desempenho na condição Neutra (sem diferença de contraste). De acordo com a predição, a acuidade global aumentou em média 7,5\% na condição Congruente e diminuiu 12,5\% na condição Incongruente. Ao contrario do estudo de Stone e Thompson (1992), nesta tarefa existia de fato uma diferença de velocidade entre os dois PLW. Dado que este estudo envolveu um julgamento de velocidade verídica, o efeito de contraste parece ser ainda mais robusto. Pode-se afirmar que os efeitos de contraste não só interferem na percepção de velocidade aparente, como também na discriminação de velocidades diferentes, afetando significativamente o desempenho perceptivo. Os efeitos de contraste pronunciaram-se sobretudo para o movimento biológico, atingindo uma diferença de 20,7\% entre as condições Congruente e Incongruente. A análise dos tempos de reação veio salientar a maior exigência implicada no julgamento de velocidade de movimento biológico. Os maiores tempos de reação para esse tipo de movimento significam que os participantes requerem mais tempo para desambiguar a diferença de velocidade dos estímulos. Dado que o tempo de reação não foi afetado pela combinação velocidade/contraste, mas apenas pelo tipo de movimento, sugerie-se que o pior desempenho obtido para o movimento biológico está relacionado com as componentes espaciotemporais específicas e a consequente complexidade computacional relacionada com o estímulo biológico.

\section{Experiência 2}

O tipo e o tempo de apresentação do estímulo podem ser pontos de critica aos resultados obtidos na Experiência 1. Primeiro, a diferença óbvia de complexi- 
dade entre o movimento em translação rígido e o biológico pode ser responsável pelas diferenças claras de resultados, com pior desempenho perante o movimento biológico. Contudo, a percepção de movimento biológico em translação pode ser um processo mais robusto, quando comparado com outros padrões complexos. Segundo, a análise dos tempos de reação demonstrou uma clara diferença entre a percepção do movimento em translação rígido e o biológico, já que o último necessita de um maior período de tempo para ser processado. O tempo de resposta médio perante o movimento rígido coincidiu com o tempo de apresentação (1s), enquanto o tempo de resposta médio para o movimento biológico coincidiu já com o intervalo interestímulo. Esses dados podem indicar que o tempo de apresentação, no que diz respeito ao movimento biológico, é demasiado curto para permitir uma adequada integração do movimento biológico em translação.

O objetivo da Experiência 2 foi testar os pontos indicados anteriormente. A nova tarefa experimental foi desenvolvida com o intuito de verificar se é obtido melhor desempenho perceptivo na presença de movimento biológico canônico, quando este é comparado com outros padrões de movimento complexos. Assim, dois padrões de movimento complexo foram comparados: estímulos de movimento em translação biológico canônico e biológico invertido. Na literatura, o PLW invertido é considerado como um estímulo de movimento complexo, idêntico ao movimento biológico canônico em termos de informação da forma e estrutura, mas significativamente diferente em termos da percepção de eventos dinâmicos (Shipley, 2003). O reconhecimento de PLW decresce de forma significativa durante a apresentação de padrões de movimento biológico invertidos (Pavlova \& Sokolov, 2000; Sumi, 1984).

Com base nos resultados do tempo de reação obtidos para o movimento biológico na Experiência 1, o tempo de apresentação dos estímulos da Experiência 2 foi alargado para 1.5s, já que todos os estímulos são complexos.

\section{Participantes}

Cinco participantes realizaram a tarefa experimental deste estudo. Todos possuíam visão normal ou corrigida, confirmada pelo teste automático Ergovision
(Essilor) e por testes complementares de cinética (acuidade visual dinâmica, com estímulos em movimento cuja direção, velocidade e frequência espacial variavam) e visão de contraste.

\section{Instrumentos}

Os equipamentos, a tarefa e o design geral utilizados na Experiência 1 foram mantidos. A combinação velocidade/contraste e o tipo de movimento mantiveram-se como fatores de análise. Os tipos de movimento apresentados nessa tarefa foram o movimento em translação biológico canônico e o invertido. O estímulo biológico canônico foi o mesmo utilizado na Experiência 1. O estímulo biológico invertido foi criado a partir da rotação de $180^{\circ}$ do eixo dos X do mesmo PLW. A componente de translação foi mantida. Relativamente à combinação velocidade/contraste, e dado que foram obtidas diferenças significativas apenas entre as condições Congruente e Incongruente, essas duas condições foram mantidas. A condição Neutra foi eliminada do design experimental. O tempo de apresentação dos estímulos foi alargado para 1.5s, de forma a acomodar o tempo de reação mais longo requerido pelo movimento biológico canônico na primeira experiência.

\section{Procedimentos}

As instruções e procedimentos experimental foram exatamente os mesmos da Experiência 1.

\section{Resultados}

A análise de dados exploratória revelou a ausência de qualquer viés de posição, ou seja, não foi encontrada preferência pela posição dos PLW, seja inferior (51\%), seja superior (49\%). Todos os resultados apresentaram uma distribuição normal, indicada pelo teste de normalidade de Kolmogorov-Smirnov, com a correção de significância de Lilliefors. Com o intuito de verificar a ocorrência de diferenças significativas na distribuição dos dados, foram executadas análises intersujeito e item a item. Os resultados de uma one-way ANOVA demonstraram não existir efeito da variável Participante na análise de cada condição, com exceção da condição incon- 
gruente para o movimento biológico. O teste post-hoc de Scheffé indicou que o desempenho do Participante 4 foi inferior ao dos outros participantes de uma forma geral, mas não foram sinalizadas diferenças significativas nos efeitos das variáveis. Assim, foi decidido substituir o número de participantes pelo número de blocos de ensaios como unidade de análise (passou-se de $n=5$ para $\mathrm{n}=20$ ).

\section{Acuidade}

O tipo de movimento e combinação velocidade/contraste foram utilizados como fatores numa ANOVA para medidas repetidas. Os dados sugerem um efeito da combinação velocidade/contraste $[F(1,19)=$ 13.148, $p<0,05]$ e ausência de efeito do tipo de movimento $[F(1,19)=1.203$, n.s.] sobre o desempenho na tarefa. Não se verificou interação entre as duas variáveis $F(1,19)=0,834$, n.s.]. A Figura 3 ilustra a percentagem de respostas corretas para cada tipo de movimento e combinação velocidade/contraste (média +/-SD). A acuidade nos julgamentos de velocidade foi significativamente mais elevada na condição Congruente, quando comparada com a condição Incongruente. Verificou-se novamente um efeito do contraste na percepção de velocidade. O desvio-padrão (barras de erro na Figura 3) aumentou significativamente na condição Incongruente. A percentagem de respostas corretas não foi influenciada pelo tipo de movimento em translação, fosse ele biológico canônico [média(SD). 84.5(10) e 71(19), para as condições Congruente e Incongruente, respectivamente], ou invertido [média(SD). 84.5(7.5) e 75(17), para as condições Congruente e Incongruente, respectivamente].

Tempo de Reação. O tempo de reação médio não foi influenciado pelo tipo de movimento apresentado nem pela combinação velocidade/contraste, como se pode verificar na Figura 4. Os participantes levaram em média 1500ms (perto da duração do estímulo) para executar o julgamento de velocidade para os dois tipos de movimento [Movimento biológico canônico: média (SD). 1440.17(161.62) e 1484.17(140.68), para as condições Congruente e Incongruente, respectivamente; Movimento biológico invertido: média (SD). 1499(151.09) e 1564.94(163.89), para as condições Congruente e Incongruente, respectivamente]. Uma ANOVA para medidas

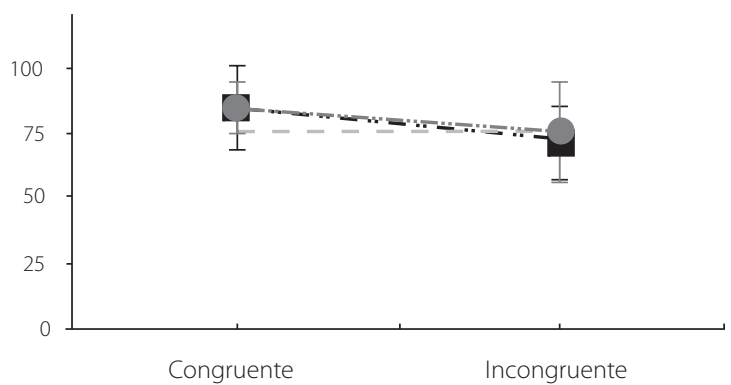

- - Biológico -.... Invertido - - - Limiar de 75\%

Figura 3. Percentagem média de respostas corretas em função da combinação Velocidade/Contraste para cada padrão de movimento, na Experiência 2. Braga (Portugal), 2008.

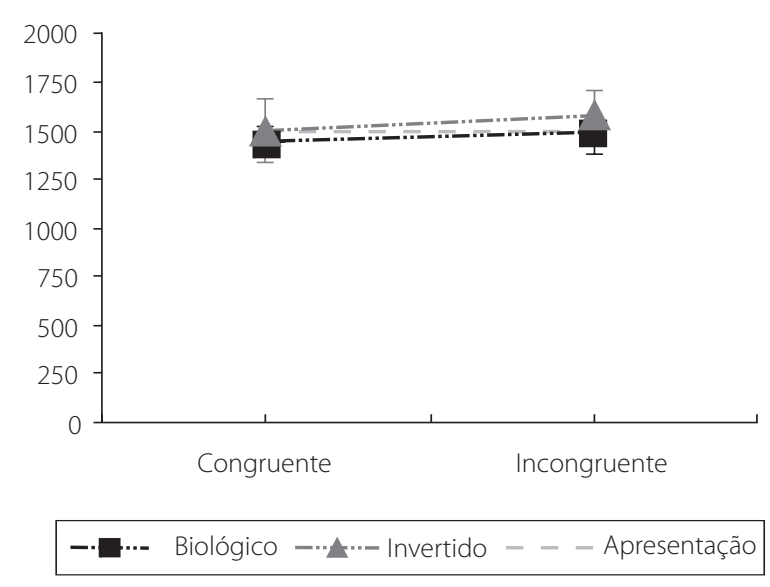

Figura 4. Tempo de reação médio (milissegundos) em função da combinação Velocidade/Contraste para cada padrão de movimento, na Experiência 2. Braga (Portugal), 2008.

repetidas demonstrou não haver efeitos do tipo de movimento $[F(1,19)=10.06$, n.s. $]$ nem da combinação velocidade/contraste $[F(1,19)=9.524$, n.s. $]$ sobre o tempo de reação.

\section{Discussão}

Os estudos clássicos de movimento biológico demonstram que um conjunto de pontos luminosos que sinalizam as articulações de uma figura humana invisível permite um reconhecimento mais robusto e mais rápido do agente e da ação, quando comparado com outro tipo de configuração complexa. Essas confi- 
gurações complexas são habitualmente PLW invertidos e scrambled (Pavlova \& Sokolov, 2000; Sumi, 1984; Troje, 2003).

No presente estudo, os participantes desempenharam o julgamento de velocidade de PLW, em movimento de translação canônico e invertido. A acuidade na tarefa não variou em função do tipo de movimento, mas foi verificado um efeito da combinação velocidade/contraste. A percentagem de respostas corretas foi superior, de uma forma estatisticamente significativa, na condição Congruente. A diminuição da qualidade do desempenho foi acompanhada por um aumento da incerteza. Esse efeito verificou-se para ambos os movimentos em translação, tanto o biológico canônico quanto o invertido.

Nessa tarefa, a presença de um padrão familiar de movimento pareceu não promover o funcionamento de um mecanismo específico para o movimento biológico, pelo menos no que diz respeito à percepção de velocidade. Além disso, não foram verificadas diferenças no tempo de reação para os dois tipos de movimento. Desse modo, pode-se sugerir que as diferenças entre o estímulo biológico canônico e o invertido têm efeitos em tarefas de reconhecimento e discriminação de direção, mas não na percepção de padrões de movimento em translação. Além disso, o aumento no tempo de apresentação do estímulo (de 1s para 1.5s) não promoveu nenhuma diferença clara no desempenho da tarefa entre a Experiência 1 e 2, relativamente ao movimento biológico canônico. Portanto, o pobre desempenho obtido para o movimento biológico em translação, quando comparado com o movimento rígido, não se deve ao curto tempo de apresentação, mas sim à diferença de complexidade do estímulo.

\section{Experiência 3}

A manutenção do mesmo ponto de partida da trajetória dos PLW entre os ensaios, como ocorreu nas experiências anteriores, pode funcionar como uma pista espacial para o julgamento de velocidade. Dado que foi mantido o mesmo ponto de partida para todas as condições experimentais, as diferenças obtidas não se devem a esse fato. Mesmo assim, para controlar o papel da pista de posição no desempenho da tarefa, na Experiência 3 os pontos inicial e final da trajetória foram variados, de forma a que tanto o PLW mais rápido como o mais lento pudessem terminar o movimento num ponto mais avançado ou mais atrasado da trajetória. 0 objetivo da implementação desse procedimento foi verificar se o desempenho na tarefa baseou-se na simples deslocação espacial do PLW, ou se foi efetuado de fato um julgamento de velocidade.

\section{Participantes}

Nove participantes realizaram a tarefa experimental deste estudo, três em cada uma das subtarefas. nenhum dos participantes conhecia o enquadramento e objectivos experimentais. Os participantes possuíam visão normal ou corrigida, confirmada pelo teste automático Ergovision (Essilor) e por testes complementares de cinética e visão de contraste.

\section{Instrumentos}

Os equipamentos, a tarefa e o design geral utilizados nas experiências anteriores foram mantidos. A combinação velocidade/contraste e o tipo de movimento mantiveram-se como fatores de análise. Todos os tipos de movimento apresentados nas experiências anteriores foram utilizados nesta tarefa: movimento em translação rígido, biológico canônico e invertido. As combinações velocidade/contraste foram apresentadas independentemente em três subtarefas: $3 a$ - Condição Incongruente com contraste de Weber de 99; 3b-Condição Incongruente com contraste de Weber de 29 ( 2 and $\left.60 \mathrm{~cd} / \mathrm{m}^{2}\right) ; 3 \mathrm{c}$ - Condição Congruente com contraste de Weber de 29.

Em cada subtarefa foi aplicado um design $3 * 1$ (padrão de movimento* ${ }^{*}$ combinação contraste/velocidade). Cada subtarefa representa um nível de exigência diferente. A diminuição dos efeitos de contraste, i.e., tornando o efeito de contraste mais sutil, pode salientar diferenças de desempenho entre os diferentes tipos de movimento. O objetivo dessa variação foi controlar alguns efeitos de teto e chão, verificados para o movimento rígido na condição Congruente, e para o movimento biológico na condição Incongruente, respectivamente. Os efeitos de teto e chão obtidos nas experiências anteriores podem estar mascarando possíveis diferenças entre os tipos de movimento. 


\section{Procedimentos}

As instruções e os procedimentos experimental foram exatamente os mesmos da Experiência 1.

Foram apresentados seis blocos de quinze ensaios para cada um dos três padrões de movimento, resultando em 270 ensaios para cada uma das subtarefas. Os blocos foram apresentados numa ordem quase aleatória. O tempo de apresentação do estímulo foi de $1.5 \mathrm{~s}$, e os pontos inicial e final variaram ao longo da trajetória do PLW. A manutenção da mesma posição de partida do PLW em todos os estímulos poderia resultar na utilização de uma pista artefactual na discriminação de velocidade. Com o intuito de reduzir esse tipo de pista na experiência, optou-se por variar os pontos de partida e chegada, fazendo com que tanto o PLW mais lento como o mais rápido pudessem terminar o ensaio na posição relativa mais avançada ou atrasada. Assim garantiu-se uma tarefa baseada no julgamento de velocidade, e não na diferença de distâncias.

\section{Resultados}

A análise de dados exploratória revelou a ausência de missing values e de qualquer viés de posição, ou seja, não foi encontrada preferência pela posição dos PLW, nem inferior nem superior. Com o intuito de verificar a ocorrência de diferenças significativas na distribuição dos dados, foram executadas análises intersujeito e item a item. Dado que o desvio-padrão e a variância dos dados não variaram entre os sujeitos para cada um dos itens, foi decidido substituir o número de participantes pelo número de blocos de ensaios como unidade de análise (passou-se de $\mathrm{N}=3$ para $\mathrm{N}=18$ em cada uma das subtarefas).

Subtarefa 3a. Nesta subtarefa foi apresentada a condição Incongruente com o nível de contraste de 99, para os três tipos de movimento. Para o movimento rígido, a percentagem média de respostas corretas foi de 82.0 (DP=23.1) - acima do limiar de 75\%. De outro lado, a percentagem média de respostas corretas diminuiu para valores próximos do acaso (50\%) para ambos os padrões de movimento em translação biológico, tanto o canônico quanto o invertido (média (DP). 53,9(26.0) e 55,65(26.7), respectivamente), como pode ser observado na Figura 5. A ANOVA para medidas repetidas revelou um forte efeito do fator padrão de movimento. O declínio no desempenho entre o padrão de movimento em translação rígido e os outros dois é estatisticamente significativo $[F(1.02,25.5)=17.56 ; p<0,001]$. Uma análise Pairwise, utilizando o ajuste de Bonferroni para múltiplas comparações, não encontrou diferenças significativas na discriminação de velocidade entre os padrões de movimento em translação biológico canônico e invertido.

Os baixos valores de desempenho para os padrões de movimento em translação biológico canônico e invertido podem ter atenuado possíveis diferenças no desempenho para esses dois padrões de movimento. As duas tarefas subsequentes visaram explorar tal questão, através da introdução de condições experimentais menos exigentes. Para isso, o nível de contraste mínimo entre o PLW e o fundo diminuiu. Esse procedimento teve como principal objetivo diminuir a dificuldade da tarefa, de modo a evitar padrões de resposta próximos dos valores do acaso. Ao mesmo tempo, permitiu verificar se a magnitude da interferência do fundo varia conforme a diferença de contraste.

Subtarefa 3b. A metodologia experimental da subtarefa anterior foi mantida, mas o nível de contraste entre o PLW e o fundo diminuiu para um nível de 29, mantendo-se a condição Incongruente. O efeito da combinação contraste/velocidade diminuiu e, consequentemente, o desempenho médio na tarefa aumentou para os padrões de movimento rígido [média(DP). 83.7(19.0)], biológico canônico [média(DP). 65.9(23.0)] e biológico invertido [média(DP). 62.2(23.1)], como pode ser observado na Figura 5. Mesmo assim, as diferenças no desempenho da tarefa experimental para os três padrões de movimento são congruentes com os resultados da subtarefa anterior. Uma ANOVA de medidas repetidas voltou a revelar um forte efeito para o fator padrão de movimento. A discriminação de velocidade de estímulos com movimento em translação rígido é significativamente superior, quando comparada com os outros padrões de movimento em translação [F $(5.5,93.5)=6.46, p<0,005]$. Uma análise Pairwise, utilizando o ajuste de Bonferroni para múltiplas comparações, não encontrou diferenças significativas na discriminação de velocidade entre os padrões de movimento em translação biológico canônico e invertido.

Subtarefa 3c. Na subtarefa anterior, a percentagem de respostas corretas voltou a ser baixa para os padrões 
A

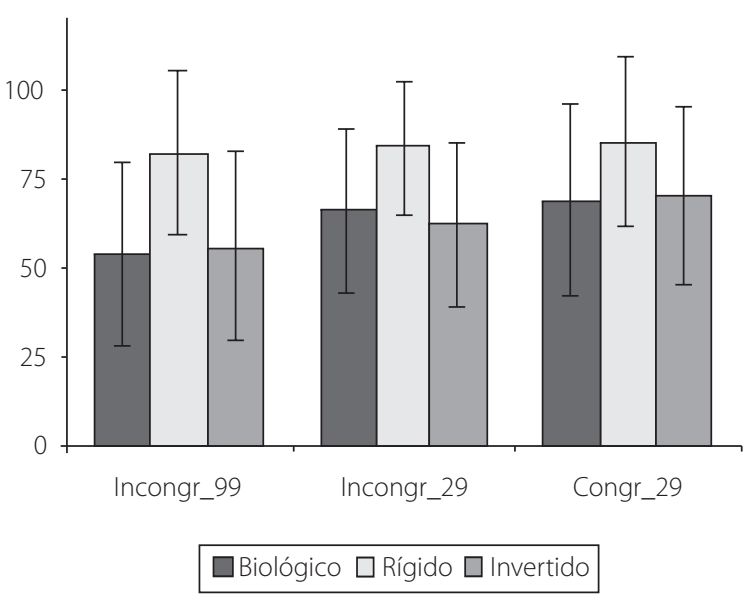

B

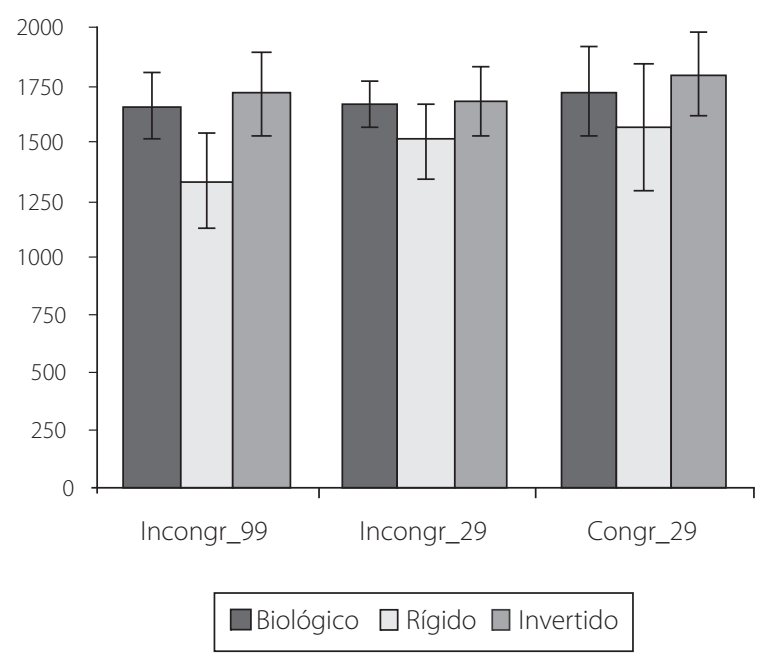

Figura 5. Experiência 3 (A) Percentagem média de respostas corretas para cada padrão de movimento e combinação velocidade/contraste. (B) Tempo de reação médio (milissegundos) para tipo de movimento e combinação velocidade/contraste. Barras de erro representam o desvio padrão. Braga (Portugal), 2008.

de movimento em translação biológico canônico e invertido, não tendo chegado ao limiar dos 75\%. Por isso, na subtarefa 3c, a exigência da tarefa experimental foi uma vez mais reduzida, através da utilização da condição Congruente (PLW mais rápido com maior contraste com o fundo), com um nível de contraste de 29.

486 Os resultados indicaram um aumento no desempenho global da tarefa, tal como esperado (Figura 5) e, mais uma vez, o padrão de resultados foi semelhante ao das subtarefas anteriores. Para o movimento rígido atingiram-se valores superiores ao limiar de 75\% [média(DP). 85.6(24.2)]. Para os restantes padrões de movimento em translação, a percentagem média de respostas corretas foi significativamente inferior (média(DP). 68.9(26.5) para o movimento biológico canônico; média(DP). 70.0(25.2) para o movimento biológico invertido). A ANOVA de medidas repetidas revelou um forte efeito para o fator padrão de movimento [F $(.452,7.69)=4.96, p<0,05]$. Uma análise Pairwise, utilizando o ajuste de Bonferroni para múltiplas comparações, não encontrou diferenças significativas na discriminação de velocidade entre os padrões de movimento em translação biológico canônico e invertido. Além disso, as pequenas variações nos desempenhos não são consistentes entre os padrões de resposta individuais, ou seja, em algumas situações o desempenho é mais elevado para o movimento em translação biológico canônico, e noutras para o invertido.

Tempo de reação. Os dados relativos ao tempo de reação das três subtarefas são agora apresentados. Como pode ser observado na Figura 5, verificou-se um efeito principal do padrão de movimento sobre o tempo de reação. Relativamente ao padrão de movimento translacional rígido, o tempo de reação médio de 1500ms (DP. 240) coincidiu com o tempo de apresentação do estímulo, enquanto para os padrões de movimento translacional biológico canônico [média(DP). de 1670ms(137)] e invertido [média(DP). 1710ms(165)], o tempo de reação foi significativamente superior [F $(1.12,13.42)=35.05, p<0,01]$. O teste post hoc de Schefée demonstrou que as diferenças no tempo de reação são significativas entre o movimento rígido e os outros dois movimentos, mas não existem diferenças significativas entre os movimentos em translação biológico canônico e invertido. Não existem diferenças significativas entre as diferentes combinações contraste/velocidade [F $(2,34)=2.44$, n.s.].

\section{Discussão}

A análise integrada dos resultados obtidos nas três subtarefas demonstrou um padrão de resultados semelhante ao encontrado nas experiências anteriores. 
A acuidade na tarefa decresceu na condição Incongruente, quando comparada com a condição Congruente. Na subtarefa 3a, foi apresentado um estímulo Incongruente com um alto nível de contraste. Nessa condição, o efeito de contraste atingiu a maior magnitude. Na subtarefa 3b, o nível de contraste foi reduzido e a condição Incongruente foi mantida, o que originou um aumento do desempenho. Esse dado revela que a interferência do fundo na percepção de velocidade é um efeito quantificável e variável tendo em conta o nível de contraste, o que é suportado pelos resultados de Stone e Thompson (1992). A subtarefa 3c é representativa do menor nível de exigência do julgamento de velocidade. Nessa tarefa, o desempenho para os movimentos biológico canônico e invertido sofreu um aumento significativo, tendo atingido valores próximos dos 70\%. Mesmo assim, não se verificaram diferenças entre esses padrões de movimento.

Quanto à comparação do efeito da variável padrão de movimento na realização das três subtarefas experimentais, verificou-se um desempenho significativamente mais elevado nos ensaios em que foi apresentado o padrão de movimento em translação rígido. Verificou-se efeito de contraste para todos os padrões de movimento analisados, com maior magnitude para os padrões mais complexos, quais sejam o biológico canônico e o invertido. A hipótese de maior exigência computacional por parte dos padrões de movimento biológico canônico e invertido foi assim suportada por esses resultados. Ao contrário dos estudos de máscara no reconhecimento de movimento biológico, os resultados obtidos neste estudo sugerem não existir especial resistência desse padrão à interferência do fundo, pelo menos em tarefas que envolvam percepção de velocidade. A análise dos tempos de reação salienta a dificuldade verificada no desempenho da tarefa para os movimentos complexos. O fato de o tempo de reação obtido para os padrões de movimento em translação biológico canônico e invertido ser significativamente mais elevado do que para o movimento rígido, é explicado pelo maior nível de exigência imposto pelos padrões computacionalmente mais complexos.

Os dados apresentados demonstram que a magnitude da interferência do fundo varia com o nível de contraste e com a combinação contraste/velocidade, de uma forma proporcional. Outro dado importante é que, com a manipulação dos pontos de partida e de chegada (diferentes entre ensaios e velocidades), verificou-se que os participantes baseiam seu desempenho no julgamento de velocidade, e não na utilização de pistas espaciais de distância percorrida.

\section{Considerações Finais}

Como em estudos anteriores os resultados obtidos nesta pesquisa revelam efeitos de contraste sobre a percepção de velocidade. Referem que, quando o contraste de um objeto de referência (teste) numa discriminação de velocidade é mais elevado, a velocidade do outro objeto é subestimada em cerca de 14\%. Por outro lado, quando o contraste do objeto de teste é menor, a velocidade do outro objeto é sobrestimada em cerca de $17 \%$. Esses valores foram encontrados para estímulos apresentados simultaneamente com a mesma velocidade, daí o julgamento ser baseado na velocidade aparente.

No presente estudo, em todos os ensaios existia uma diferença efetiva de velocidade. Isso significa que, verificando-se um efeito de contraste, a deterioração no desempenho na condição Incongruente (o PLW mais rápido com menor contraste), relativamente à condição Congruente (o PLW mais rápido com maior contraste), foi um fenômeno ainda mais robusto que o reportado anteriormente. A interferência do fundo afeta não só a velocidade aparente, como também a percepção de diferenças de velocidade verídica.

No que diz respeito à percepção de velocidade, não foi encontrada nenhuma especialização do sistema visual, nem dependência da perspectiva para o movimento biológico. Os resultados são independentes da familiaridade do estímulo; portanto, a discriminação de velocidade baseia-se na comparação de configurações de pontos, sem relação com seu significado ou identificação. Tais resultados, mais uma vez, contrariam os estudos clássicos de reconhecimento e identificação de movimento biológico.

Em suma, verificou-se que o movimento translacional biológico é bastante sensível à interferência do fundo, pelo menos no que diz respeito aos efeitos de contraste na percepção de velocidade. Concluiu-se também que a familiaridade do padrão não é crucial para o desempenho de tarefa. Esses dados, bem como o desempenho mais pobre na presença do movimento 
biológico quando comparado com o rígido, diferenciam-se das conclusões da maioria dos estudos clássicos de reconhecimento de movimento biológico.

\section{Referências}

Anstis, S. (2001). Footsteps and inchworms: illusions show that contrast affects apparent speed. Perception, 30, 785-794.

Anstis, S. (2003). Moving objects appear to slow down at low contrasts. Neural Networks, 16, 933-938.

Atkinson, A. P., Dittrich, W. H., Gemmell, A. J., \& Young, A. W. (2004). Emotion perception from dynamic and static body expressions in point-light and full-light displays. Perception, 33, 717-46.

Beardsworth, T., \& Buckner, T. (1981). The ability to recognize oneself from a video recording of one's movements without seeing one's body. Bulletin of the Psychonomic Society, 18, 19-22.

Bertenthal, B. I., \& Pinto, J. (1994). Global processing of biological motions. Psychological Science, 5, 221-225.

Cutting, J. E., Moore, C., \& Morrison, R. (1988). Masking the motions of human gait. Perception \& Psychophysics, 44 (4), 339-347.

Dekeyser, M., Verfaillie, K., \& Vanrie, J. (2002). Creating stimuli for the study of biological-motion perception. Behavior Research Methods, Instruments \& Computer, 34 (3), 375-382.

Dittrich, W. H. (1993). Actions categories and the perception of biological motion. Perception, 22, 15-22.

Johansson, G. (1973). Visual perception of biological motion and a model for its analysis. Perception \& Psychophysics, 14, 201-211.

Neri, P., Morrone, M. C., \& Burr, D. C. (1998). Seeing biological motion. Nature, 395, 894-896.

Norman, J. F., Payton, S. M., Long, J. R., \& Hawkes, L. M. (2004). Aging and perception of biological motion. Psychological Aging, 19, 219-225.
Pavlova, M., \& Sokolov, A. (2000). Orientation specificity in biological motion perception. Perception \& Psychophysics, 62 (5), 889-899.

Peli, E. (1990). Contrast in Complex Images. Journal of the Optical Society of America, 7 (10), 2032-2040.

Pollick, F. E., Paterson, H. M., Bruderlin, A., \& Sanford, A. J. (2001). Perceiving affect from arm movement. Cognition, 82, B51-B61.

Pollick, F. E., Kay, J. W., Heim, K., \& Stringer, R. (2005). Gender recognition from point-light walkers. Journal of Experimental Psychology:Human Perception and Performance, 31, 1247-1265.

Shipley, T. F. (2003). The effect of object and event orientation on perception of biological motion. Psychological Science, 14 (4), 377-380.

Stone, L. S., \& Thompson, P. (1992). Human speed perception is contrast dependent. Vision Research, 32 (8), 1535-1549.

Sumi, S. (1984). Upside-down presentation of the Johansson moving light-spot pattern. Perception, 13 (3), 283-286.

Thompson, B., Hansen, B. C., Hess, R. F., \& Troje, N. F. (2007). Peripheral vision: good for biological motion, bad for signal noise segregation? Journal of Vision, $7(10), 1-7$.

Thornton, I., Pinto, J., \& Shiffrar, M. (1998). The visual perception of human locomotion across space and time. Cognitive Neuropsychology, 15, 535-552.

Troje, N. F. (2003). Reference frames for orientation anisotropies in face recognition and biological-motion perception. Perception, 32 (2), 201-10.

Troje, N. F., Sadr, J., Geyer, H., \& Nakayama, K. (2006). Adaptation aftereffects in the perception of gender from biological motion. Journal of Vision, 6 (8), 850-857.

Westhoff, C., \& Troje, N. F. (2007). Kinematic cues for person identification from biological motion. Perception \& Psychophysics, 69, 241-253.

Recebido em: 5/3/2009

Versão final reapresentada em: 4/7/2011

Aprovado em: 9/8/2011 